\title{
Analytical approximation of the soliton solutions of the quintic complex Ginzburg-Landau equation
}

\author{
J. M. Soto-Crespo \\ Instituto de Óptica, CSIC, Serrano 121, 28006 Madrid, Spain \\ Luis Pesquera \\ Facultad de Ciencias, Instituto de Física de Cantabria, CSIC-UC, Avenida Los Castros s/n, E-39005 Santander, Spain
}

(Received 30 June 1997)

\begin{abstract}
We have performed a theoretical study of the soliton fiber laser based on the quintic complex GinzburgLandau equation (CGLE). This study may also apply to soliton propagation in telecommunications systems. We have developed a simple approach that allows us to obtain, in an approximate way, analytical expressions for the stable pulselike solutions of the CGLE. The method also gives an accurate estimate of the region in the parameter space where stable pulselike solutions exist. We also obtain that the minimum allowed value of the peak amplitude of the soliton solutions depends solely on the relation between the linear loss term and the quintic gain saturation term. The predictions are confirmed by numerical simulations.
\end{abstract}

[S1063-651X(97)11112-6]

PACS number(s): 42.65.Tg, 47.20.Ky, 42.55.Wd

\section{INTRODUCTION}

Soliton generation in actively and passively mode locked fiber lasers [1-4] is presently a very active area of research. Fiber lasers with additive-pulse mode locking or nonlinear polarization rotation [5-17] may be described by the complex Ginzburg-Landau equation (CGLE). This equation also applies when describing soliton propagation in optical fiber systems with linear and nonlinear gain and spectral filtering (such as communication links with lumped fast saturable absorbers [18-25]). Different forms of the CGLE have been used, including the cubic Ginzburg-Landau equation [26], cubic CGLE with saturation [7,9], quintic CGLE $[5,22,24,27,28]$, and more complicated models.

The quintic CGLE can be written in the form $[24,27]$

$$
\begin{gathered}
i \psi_{z}+\frac{D}{2} \psi_{t t}+|\psi|^{2} \psi= \\
+i \delta \psi+i \epsilon|\psi|^{2} \psi+i \beta \psi_{t t}-\nu|\psi|^{4} \psi \\
+i \mu|\psi|^{4} \psi
\end{gathered}
$$

where $z$ is the propagation distance, $t$ is the retarded time, $\psi$ is the normalized envelope of the electric field, $D= \pm 1$ is the chromatic dispersion coefficient, $\beta$ stands for spectral filtering $(\beta>0), \delta$ is the linear gain or loss coefficient, $\epsilon$ and $\mu$ represent nonlinear gain or loss (which describe, for instance fast saturable absorption), and $\nu$ is the saturation of the nonlinear refractive index. As long as $\nu$ is small $(\mid \nu$ $\mid \ll 1)$ its influence on the formation of stable pulselike solutions is neglectable [29]; therefore, we shall consider $\nu=0$ in the rest of the paper. The other quintic term being negative $(\mu<0)$ is generally, although not always [30], necessary for providing stability against the infinite growth expected from the cubic gain $(\epsilon>0)$.

As is well known, in the anomalous dispersion regime $(D=+1)$ bright soliton solutions of the nonlinear Schrödinger equation (NLSE) exist. In this case, for very small values of the coefficients on the right-hand side of Eq. (1), the solitary wave solutions of the CGLE with $D=+1$ are close to the usual NLSE solitons. As a consequence, in the anomalous dispersion regime it is possible to study the main properties of the solitonlike solutions of Eq. (1) by applying the well-developed soliton perturbation theory of the NLSE $[31,32]$. This approach, however, cannot be used with normal dispersion [i.e., $D=-1$ in Eq. (1)], since in this case no solution of the NLSE in the form of a bright pulse exists. Furthermore, perturbation theory cannot be applied when $|\delta|$, $\beta,|\epsilon|,|\nu|$, and $|\mu|$ are not much less than 1 .

The solitary wave solutions of the CGLE and their stability have been analyzed recently in previous papers for the cases of anomalous [28,29] and normal dispersion [33]. Analytical, exact pulselike solutions of the quintic CGLE have been found in a few special cases and in general they are unstable. On the other hand, stable pulselike solutions have been found numerically. Their existence for a wide interval of values of the parameters of the quintic CGLE has been shown recently $[29,33]$. However, numerical studies give only a partial picture of the problem as they must be conducted for a few values of the equation parameters. In addition, quite often one needs to fit the experimental data to an expected pulselike solution, which cannot be done when no analytical expression exists for it. Starting from the stable pulselike solutions of the cubic CGLE for $D=+1$, which have an analytical expression, in Sec. II we relate them to the stable pulselike solutions of the quintic CGLE and this allows us to find an approximated expression for them. An expression for the lower boundary of the stability region in the plane $(\beta, \epsilon)$ is obtained. The minimum allowed value of the peak amplitude of the soliton solutions is found to depend exclusively on $\delta / \mu$ and therefore is independent of $\beta$ and $\epsilon$. We then extend the approach to the case of normal dispersion. The analytical predictions of our approach are verified in Sec. III. Finally, Sec. IV summarizes the main results. 


\section{ANALYTICAL APPROXIMATION}

We first recall that the cubic CGLE [i.e., Eq. (1) with $\mu=\nu=0$ ] possesses stable pulselike solutions only for $D=+1, \delta=0$, and $\epsilon$ and $\beta$ related in the following way:

$$
\epsilon=\epsilon_{s}=\beta \frac{3 \sqrt{1+4 \beta^{2}}-D}{4+18 \beta^{2}}
$$

[The above equation defines a line in the plane $(\beta, \epsilon)$ that we shall call from now on line $S$.] This solution is given by [28]

$$
\Psi_{0}(t, z)=A \operatorname{sech}(B t) \exp \{i d \ln [\operatorname{sech}(B t)]\} \exp (-i \omega z),
$$

where $\mathrm{A}$ is an arbitrary positive parameter, and $d, \omega$ and $A / B$ are given by

$$
\begin{gathered}
d=\frac{\sqrt{1+4 \beta^{2}}-D}{2 \beta}, \quad \omega=-d \frac{1+4 \beta^{2}}{2 \beta} B^{2}, \\
\frac{A}{B}=\sqrt{\frac{\left(2+9 \beta^{2}\right) \sqrt{1+4 \beta^{2}}\left(\sqrt{1+4 \beta^{2}}-D\right)}{2 \beta^{2}\left(3 \sqrt{1+4 \beta^{2}}-D\right)}} .
\end{gathered}
$$

Equation (2) divides the plane $(\beta, \epsilon)$ into two parts. Generally speaking, for $(\beta, \epsilon)$ above the line $S$, input pulses grow indefinitely; below that line, spectral filtering dominates over the nonlinear gain and pulses decay when propagating. The presence of the quintic term saturating the nonlinear gain $(\mu<0)$ avoids the problem for points above the line $S$ and stable pulselike solutions are obtained in a wide region [29,33], when $\delta \leqslant 0$ (which stabilizes the background).

We now assume that these stable solutions (more specifically, those called plain pulses [34]) belong to the same family as the solutions given by Eq. (3), having approximately the same form, and that the fact that $\delta, \epsilon-\epsilon_{s}$, and $\mu$ are different from zero uniquely determines the value of the amplitude $A$. Naturally, this approximation will be more accurate as $\epsilon-\epsilon_{s}, \delta$, and $\mu$ tend to zero.

Introducing Eq. (3) into Eq. (1), one obtains

$$
\left(\epsilon-\epsilon_{s}\right)\left|\Psi_{0}(t)\right|^{2}+\mu\left|\Psi_{0}(t)\right|^{4}+\delta=0
$$

[where we used the definition $\Psi_{0}(t, z)=\Psi_{0}(t) \exp (-i \omega z)$ ]. Equation (6) cannot ever be satisfied for all values of $t$. Nevertheless, as long as $\left|\epsilon-\epsilon_{s}\right|, \mu$, and $\delta$ are kept small, expression (6) is quite close to zero. In our approach we consider that Eq. (6) is satisfied when averaging in time; for this task we choose the density of probability

$$
p(t)=\frac{\left|\Psi_{0}(t)\right|^{2}}{\int_{-\infty}^{\infty}\left|\Psi_{0}(t)\right|^{2} d t} .
$$

It should be noted that this density of probability makes the rate of change of energy with respect to $z$ zero, a condition that must be fulfilled for any stationary solution [35], i.e.,

$$
\begin{aligned}
\frac{d}{d z} \int_{-\infty}^{\infty}|\psi|^{2} d t & =2 \int_{-\infty}^{\infty}\left[\delta|\psi|^{2}+\epsilon|\psi|^{4}+\mu|\psi|^{6}-\beta\left|\psi_{t}\right|^{2}\right] d t \\
& =0 .
\end{aligned}
$$

Multiplying $p(t)$ by Eq. (6) and integrating in time, we easily find the following expression for $A^{2}$ :

$$
A^{2}=\frac{-5\left(\epsilon-\epsilon_{s}\right)-5 \sqrt{\left(\epsilon-\epsilon_{s}\right)^{2}-24 \delta \mu / 5}}{8 \mu},
$$

which for $\delta \mu \ll\left(\epsilon-\epsilon_{s}\right)^{2}$ becomes

$$
A^{2}=-\frac{5}{4} \frac{\epsilon-\epsilon_{s}}{\mu} .
$$

Equation (9) serves also to estimate the lower limit of the region at which stable pulselike solutions exist. The discriminant must be greater than or equal to zero for the solution to exist. The minimum value of allowed $\epsilon$ becomes

$$
\epsilon_{\text {min }}=\epsilon_{s}+\sqrt{\frac{24 \delta \mu}{5}}
$$

which explains the numerical results shown in Fig. 11 of Ref. [29], namely, that in the plane $(\beta, \epsilon)$ the lower limit of the region at which stable pulselike solutions of the quintic CGLE are found is almost parallel to the line $S$ and that as $|\mu|$ or $|\delta|$ increases, this lower limit also increases.

We also obtain that there exists a minimum value for the peak amplitude, which from Eqs. (9) and (11) is found to be

$$
A_{\text {min }}^{2}=\sqrt{\frac{15}{8} \frac{\delta}{\mu}}
$$

that is, the minimum value of the peak amplitude of the soliton solutions is independent of $\beta$ and $\epsilon$, being determined uniquely by the quotient $\delta / \mu$. Note that Eq. (12), in combination with Eq. (5), gives the allowed values of the peak amplitude and the width of the stable soliton solutions.

\section{NUMERICAL RESULTS}

In this section we compare the approximated results given by our approach with the exact ones found numerically following the method described in Ref. [29]. We have concentrated our efforts on the anomalous dispersion regime where the solutions of arbitrary amplitude [Eq. (3)] are stable. We found excellent agreement between the approximated and the exact results. In addition, the predictions of our approximation are confirmed by the numerical experiments. Finally, we check the validity of our approximation for the case of normal dispersion.

\section{A. Anomalous dispersion regime}

Figure 1(a) shows a comparison between the peak intensity for the exact pulselike solutions of the quintic CGLE found numerically following the method described in Ref. [29] and the values predicted by Eqs. (9) and (10) vs $\epsilon$. The dotted lines are for Eq. (10), the dashed lines for Eq. (9), and the symbols for the exact solutions. Circles are for $\beta=0.1$, 

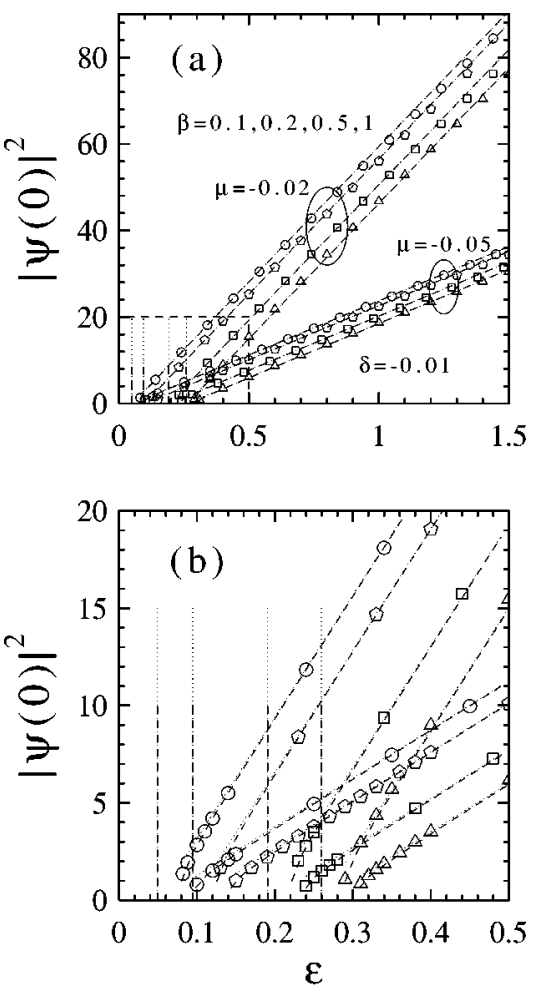

FIG. 1. Numerical (symbols) and analytical results as given by Eqs. (9) (dashed lines) and (10) (dotted lines) for the peak intensity of the solutions of the quintic CGLE vs $\epsilon$. The vertical lines are for $\epsilon=\epsilon_{s}$. Circles are for $\beta=0.1$, pentagons for $\beta=0.2$, squares for $\beta=0.5$, and triangles for $\beta=1$. The two sets of almost parallel lines correspond to $\mu=-0.02$ and -0.05 , respectively. In all the cases $\delta=-0.01$. The ellipses on the solid line embrace the two sets of curves corresponding to the two values of $\mu$ considered, which are written close to them.

pentagons for $\beta=0.2$, squares for $\beta=0.5$, and triangles for $\beta=1$. Two sets of almost parallel lines are obtained for $\mu=-0.02$ and $\mu=-0.05$ respectively as predicted by Eq. (10). Figure 1(b) shows a magnification of Fig. 1(a) around the smallest allowed values of $\epsilon$, showing the departure of the curves from a straight line. In both figures the vertical lines are for $\epsilon=\epsilon_{s}$. The matching between the exact results and the approximated ones is extraordinarily good, even for high values of $\epsilon-\epsilon_{s}$. Similarly, Fig. 2 shows the peak intensity of the stable pulselike solutions versus (a) $\mu$ and (b) $\beta$ for different values of $\epsilon$, showing an excellent coincidence between the exact results and those obtained through our approach.

Figure 3 shows (a) the amplitude and (b) phase of the exact pulselike solution and the one expressed by Eq. (3) with $A$ given by Eq. (9). The exact solution is shown as a dashed line and the approximated one as a dotted line. The values of the parameters are $\delta=-0.01, \mu=-0.05$, $\beta=0.1\left(\Leftrightarrow \epsilon_{s}=0.049\right)$, and $\epsilon=0.15,0.5$, and 0.8 . The exact and approximated pulse shapes are indistinguishable for the three values of $\epsilon$, while the phases are identical for the smallest value of $\epsilon$ and, as one would expect, the differences increase as $\epsilon-\epsilon_{s}$ increases. The phase chirp in the center of the pulse is larger than the predicted one.

The situation is similar for other values of the spectral filtering. Figure 4 is the same as Fig. 3, but for $\beta=1$ and $\epsilon=0.4,0.6$, and 0.8 . For the smallest value of $\epsilon$ the agree-
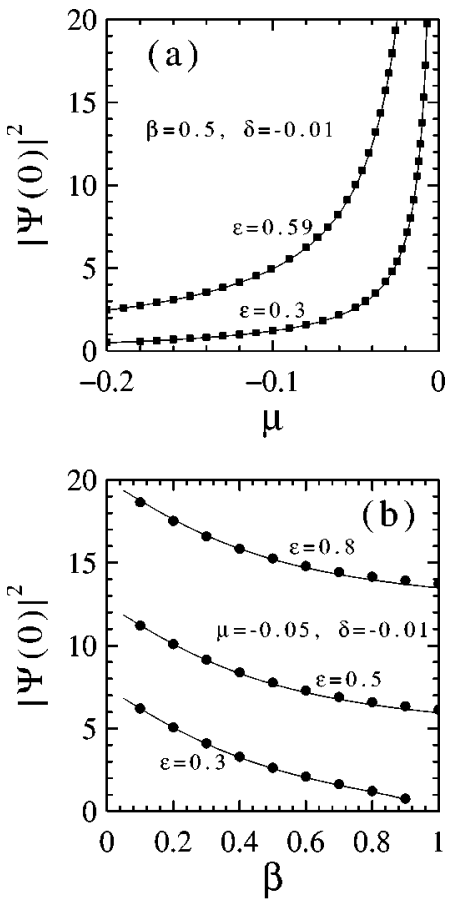

FIG. 2. Peak intensity of the stable pulselike solutions versus (a) $\mu$ and (b) $\beta$ for different values of $\epsilon$. The continuous lines stand for the results of our approximation and the filled symbols for the exact ones.
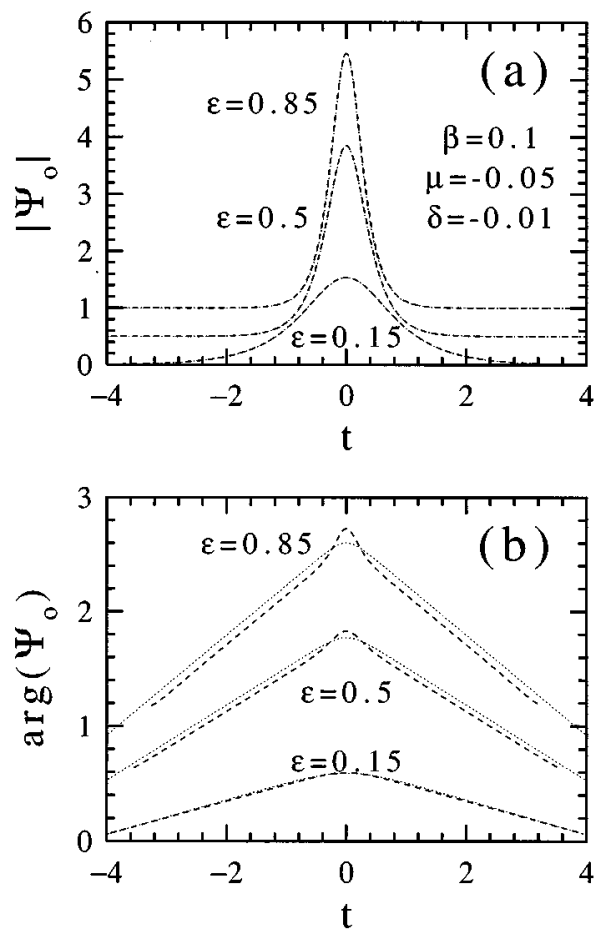

FIG. 3. (a) Field amplitude and (b) phase of the exact (dashed lines) and approximated (dotted lines) solutions of the quintic CGLE for three values of $\epsilon: 0.15,0.5$, and 0.85 . The rest of the parameters have the following values: $\beta=0.1, \mu=-0.05$, and $\delta=-0.01$. In (a) a vertical shift of 0.5 between consecutive profiles has been applied for the sake of clarity. 

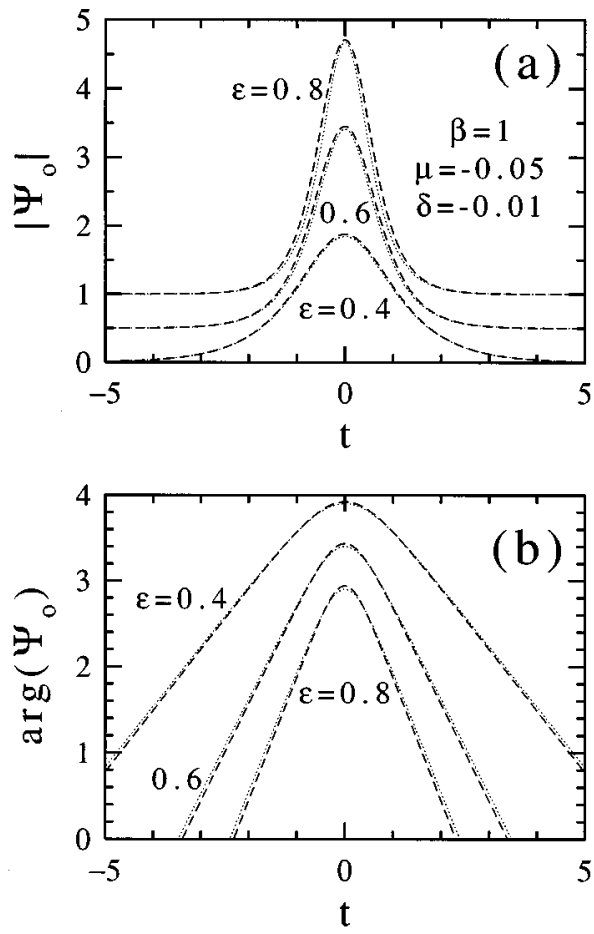

FIG. 4. Same as Fig. 3, but for $\epsilon=0.4,0.6$, and 0.8 and $\beta=1$.

ment is complete. As we increase the value of the cubic nonlinear gain the exact pulse profiles become slightly wider than the approximated ones, whereas the phases still show a remarkable coincidence.

Our approach predicts a lower boundary for the existence of soliton solutions. The qualitative agreement with previous results for $\nu$ relatively large $(\nu=0.1)$ was already mentioned above. We look now for the quantitative agreement when $\nu=0$. This limit is then given by Eq. (11). In Fig. 5 we show the line $S$ (solid line) and the above-mentioned equation for $(\delta, \mu)=(-0.01,-0.05)$ (dashed line), $(-0.05,-0.05)$ (dot-

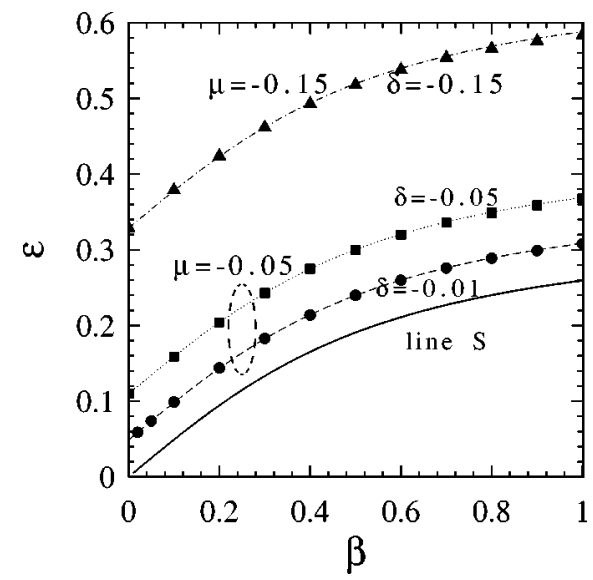

FIG. 5. The solid line corresponds to the line $S$ [Eq. (2)], the dashed, dotted, and dot-dashed lines to Eq. (11) for $(\delta, \mu)=$ $(-0.01,-0.05),(-0.05,-0.05)$, and $(-0.15,-0.15)$, respectively. The symbols are the values obtained numerically for the lower limit of the stability region of the quintic CGLE. The ellipse on the dotted line embraces the two curves corresponding to the same value of $\mu=-0.05$.

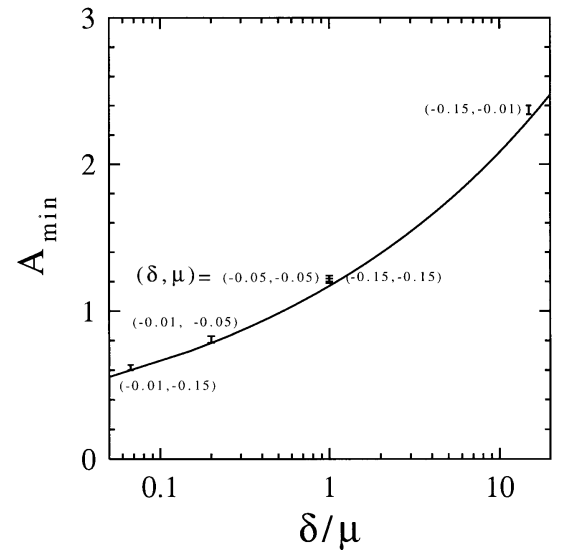

FIG. 6. Minimum value of the peak amplitude vs $\delta / \mu$ as given by Eq. (12) (solid line). The small intervals represent the numerical results obtained for the values of $(\delta, \mu)$ written close to them. The width of the intervals represents the small dispersion of $A_{\min }$ obtained when $\beta$ varies from 0 to 1 . For $\delta / \mu=1$ there are two overimposed intervals, which correspond to $\delta=-0.05$ and -0.15 , respectively.

ted line), and $(-0.15,-0.15)$ (dot-dashed line). The solid symbols represent the corresponding lower limits found numerically. We can see that even for high values of $|\delta|$ and $|\mu|$ our approach gives this limit with great accuracy. Note that the goodness of Eq. (11) could be appreciated already in Fig. 1(b): symbols representing exact solutions and dotted lines representing the approximated ones start to emerge at approximately the same values of $\epsilon$.

We finally check the minimum value of the peak amplitude as given by Eq. (12). The above results already indicate that this equation is valid. In them the value $\delta / \mu$ was around 1. We have extended the range of values of this ratio from $1 / 15$ to 15 . Figure 6 shows $A_{\min }$ vs $\delta / \mu$, as given by Eq. (12) as a solid line, and five intervals for the exact values of $A_{\min }$ calculated numerically. The intervals mean that the values of $A_{\text {min }}$ for different values of $\beta$ are inside this interval. This small dispersion in the values of $A_{\min }$ with $\beta$ could even be due to the numerical accuracy used when calculating them. In any case it is very small. Once again the approach predicts the correct pulselike solution in this very broad interval of values of $\delta / \mu$ (note the logarithmic scale on the $\mathrm{x}$ axis).

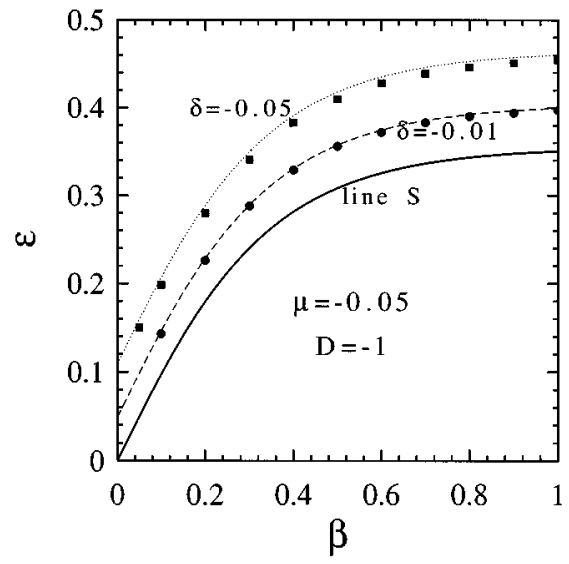

FIG. 7. Same as Fig. 5, but for normal dispersion $(D=-1)$. 

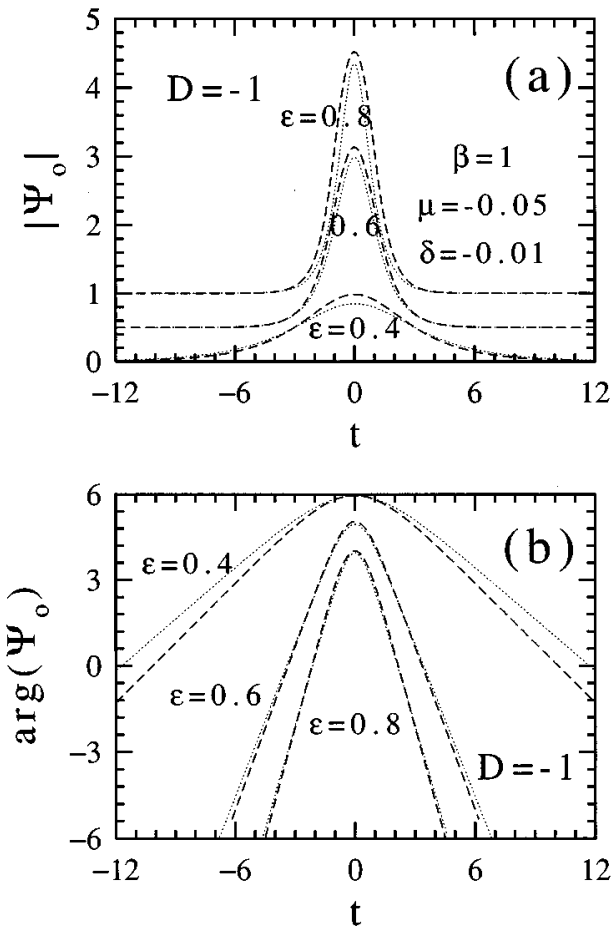

FIG. 8. Same as Fig. 4, but for normal dispersion $(D=-1)$.

\section{B. Normal dispersion regime}

In contrast to what happens in the anomalous dispersion regime, the solutions of arbitrary amplitude of the cubic CGLE as given by Eq. (3) with $D=-1$ are unstable. If we perturb them, they either grow indefinitely or continuously decay [33]. The indefinite growth can be stopped if the nonlinear gain saturates (by introducing $\mu<0$ ). All the above process can be repeated in the same way. The approximation does not work as well in the case of anomalous dispersion, but still can be helpful.

Figure 7 shows the line $S$ for normal dispersion (continuous line), the predicted lower limit [Eq. 11] for $\delta=-0.01$ (dashed line) and $\delta=-0.05$ (dotted line), and the corresponding numerical results (solid symbols). For $\beta$ close to zero, no exact pulselike solution is found, but also the analytical approach would give an infinite width. Both results are essentially the same.

However, when comparing the exact form of the field amplitude and phase of the solution with the approximated one, the agreement becomes much worse. Figure 8 is the same as Fig. 4, but for normal dispersion. Qualitatively, our approach reproduces the main features of the solution, but it predicts pulses of lower peak amplitude and smaller width than the exact ones.

\section{CONCLUSIONS}

In conclusion, we have shown that, in the anomalous dispersion regime, the stable pulselike solutions of the quintic CGLE belong to the same family of solutions as the arbitrary amplitude solutions of the cubic CGLE and therefore can be well approximated by them. The quintic term and the excess linear loss fix the unique valid amplitude for each given value of the equation coefficients. This has allowed us to obtain in an approximate way a simple analytical expression for the stable pulselike solutions of the quintic CGLE. The approximation is valid for small values of $|\delta(\leqslant 0)|$, $|\mu(<0)|$, and $\epsilon-\epsilon_{s}(>0)$. However, we find that even for large values of $\epsilon-\epsilon_{s}$ the predicted amplitude coincides with the exact one. The width and the phase chirp start to diverge before. Nevertheless, if a greater accuracy were necessary, the approximated solutions would constitute a valuable initial condition for finding the exact ones, either using a relaxation method, the shooting method, or the one used here [29].

In addition, our approach provides an accurate expression for the lower boundary of existence of soliton solutions in the plane $(\beta, \epsilon)$. This boundary is found to be a parallel curve to the line $S$ [Eq. (2)] separated from it a distance that uniquely depends on the product $\delta \mu$. In addition, the existence of a minimum peak amplitude is revealed, which depends exclusively on the relation between the linear excess loss and the quintic saturating gain term $(\delta / \mu)$. These predictions are totally corroborated by our numerical simulations and remain valid even for relative large values of $|\delta|$ and $|\mu|$. On the other hand, the relation between the peak amplitude and the width $(A / B)$ is found to depend only on the spectral filtering and on the fiber dispersion. Therefore, our results can be very useful in determining the value of system parameters required to obtain solitons with a given width and amplitude.

We have applied the approach to the normal dispersion regime, where the arbitrary amplitude solutions of the cubic CGLE are unstable. In this case the stable solutions of the quintic CGLE cannot be well approximated by them and our approach only reproduces qualitatively the main features of the stable pulselike solutions. The lower boundary of existence of soliton solutions in the plane $(\beta, \epsilon)$ is nevertheless provided with high precision.

\section{ACKNOWLEDGMENTS}

This work was supported by the Comunidad de Madrid under Contract No. 06T/039/96 and by the CICyT under Contract No. TIC95-0563. Part of this research was done while J.M.S.C was at the University of Cantabria. Their hospitality is appreciated.
[1] J. D. Kafka, T. Baer, and D. W. Hall, Opt. Lett. 14, 1269 (1989); I. N. Duling, Electron. Lett. 27, 544 (1991); Opt. Lett. 16, 539 (1991).

[2] V. J. Matsas, T. P. Newson, D. J. Richardson, and D. N. Payne, Electron. Lett. 28, 1391 (1992).
[3] S. Gray, A. B. Grudinin, W. H. Loh, and D. N. Payne, Opt. Lett. 20, 189 (1995).

[4] M. Romagnoli, S. Wabnitz, P. Franco, M. Midrio, F. Fontana, and G. E. Town, J. Opt. Soc. Am. B 12, 72 (1995).

[5] J. D. Moores, Opt. Commun. 96, 65 (1993). 
[6] O. E. Martínez, R. L. Fork, and J. P. Gordon, J. Opt. Soc. Am. B 2, 753 (1985).

[7] C. -J. Chen, P. K. A. Wai, and C. R. Menyuk, Opt. Lett. 19, 198 (1994); 20, 350 (1995).

[8] P. A. Belanger, J. Opt. Soc. Am. B 8, 2077 (1991).

[9] H. A. Haus, J. G. Fujimoto, and E. P. Ippen, J. Opt. Soc. Am. B 10, 2068 (1991).

[10] M. Hofer, M. E. Fernmann, F. Haberl, M. H. Ober, and A. J. Schmidt, Opt. Lett. 16, 502 (1991).

[11] M. Hofer, M. H. Ober, F. Haberl, and M. E. Fermann, IEEE J. Quantum Electron. 28, 720 (1992).

[12] K. Tamura, H. A. Haus, and E. P. Ippen, Electron. Lett. 28, 2226 (1992).

[13] V. J. Matsas, T. P. Newson, and M. N. Zervas, Opt. Commun. 92, 61 (1992).

[14] D. U. Noske, N. Pandit, and J. R. Taylor, Electron. Lett. 28, 2185 (1992).

[15] V. J. Matsas, D. J. Richardson, T. P. Newson, and D. N. Payne, Opt. Lett. 18, 358 (1993).

[16] H. A. Haus, E. P. Ippen, and K. Tamura, IEEE J. Quantum Electron. 30, 200 (1994).

[17] C. De Angelis, M. Santagiustina, and S. Wabnitz, Opt. Commun. 122, 23 (1995).

[18] A. Mecozzi, J. D. Moores, H. A. Haus, and Y. Lai, Opt. Lett. 16, 1841 (1991); J. Opt. Soc. Am. B 9, 1350 (1992).

[19] Y. Kodama and A. Hasegawa, Opt. Lett. 17, 31 (1992).
[20] L. F. Mollenauer, J. P. Gordon, and S. G. Evangelides, Opt. Lett. 17, 1575 (1992).

[21] L. F. Mollenauer, E. Lichtman, G. T. Harvey, M. J. Neubelt, and N. M. Nyman, Electron. Lett. 27, 792 (1992).

[22] Y. Kodama, M. Romagnoli, and S. Wabnitz, Electron. Lett. 28, 1981 (1992).

[23] H. R. Brand and R. J. Deissler, Physica A 204, 87 (1994).

[24] M. Matsumoto, H. Ikeda, T. Uda, and A. Hasegawa, J. Lightwave Technol. 13, 658 (1995).

[25] V. V. Afanasjev, Opt. Lett. 18, 790 (1993).

[26] P. A. Bélanger, L. Gagnon, and C. Paré, Opt. Lett. 14, 943 (1989).

[27] V. V. Afanasjev, Opt. Lett. 20, 704 (1995).

[28] N. N. Akhmediev, V. V. Afanasjev, and J. M. Soto-Crespo, Phys. Rev. E 53, 1190 (1996).

[29] J. M. Soto-Crespo, N. Akhmediev and V. V. Afanasjev, J. Opt. Soc. Am. B 13, 1439 (1996).

[30] W. Schöpf and L. Kramer, Phys. Rev. Lett. 66, 2316 (1991).

[31] V. I. Karpman and E. M. Maslov, Zh. Éksp. Teor. Fiz. 73, 537 (1977) [Sov. Phys. JETP 48, 281 (1978)].

[32] J. Weiland, Y. H. Ichikawa, and H. Wilhelmsson, Phys. Scr. 17, 517 (1978).

[33] J. M. Soto-Crespo, N. N. Akhmediev, V. V. Afanasjev, and S. Wabnitz Phys. Rev. E 55, 4783 (1997).

[34] V. V. Afanasjev, N. N. Akhmediev, and J. M. Soto-Crespo, Phys. Rev. E 53, 1931 (1996).

[35] N. N. Akhmediev and A. Ankiewicz, Solitons, Nonlinear Pulses and Beams (Chapman \& Hall, London, 1997). 